\title{
Low threshold, room-temperature microdisk lasers in the blue spectral range
}

\section{Citation}

Aharonovich, Igor, Alexander Woolf, Kasey J. Russell, Tongtong Zhu, Nan Niu, Menno J. Kappers, Rachel A. Oliver, and Evelyn L. Hu. 2013. Low Threshold, Room-Temperature Microdisk Lasers in the Blue Spectral Range. Applied Physics Letters 103(2): 021112.

\section{Published Version}

doi:10.1063/1.4813471

\section{Permanent link}

http://nrs.harvard.edu/urn-3:HUL.InstRepos:12111388

\section{Terms of Use}

This article was downloaded from Harvard University's DASH repository, and is made available under the terms and conditions applicable to Other Posted Material, as set forth at http:// nrs.harvard.edu/urn-3:HUL.InstRepos:dash.current.terms-of-use\#LAA

\section{Share Your Story}

The Harvard community has made this article openly available.

Please share how this access benefits you. Submit a story.

\section{Accessibility}




\section{AIP Applied Physics \\ Letters}

\section{Low threshold, room-temperature microdisk lasers in the blue spectral}

range

Igor Aharonovich, Alexander Woolf, Kasey J. Russell, Tongtong Zhu, Nan Niu et al.

Citation: Appl. Phys. Lett. 103, 021112 (2013); doi: 10.1063/1.4813471

View online: http://dx.doi.org/10.1063/1.4813471

View Table of Contents: http://apl.aip.org/resource/1/APPLAB/v103/i2

Published by the AIP Publishing LLC.

Additional information on Appl. Phys. Lett.

Journal Homepage: http://apl.aip.org/

Journal Information: http://apl.aip.org/about/about_the_journal

Top downloads: http://apl.aip.org/features/most_downloaded

Information for Authors: http://apl.aip.org/authors

\section{ADVERTISEMENT}
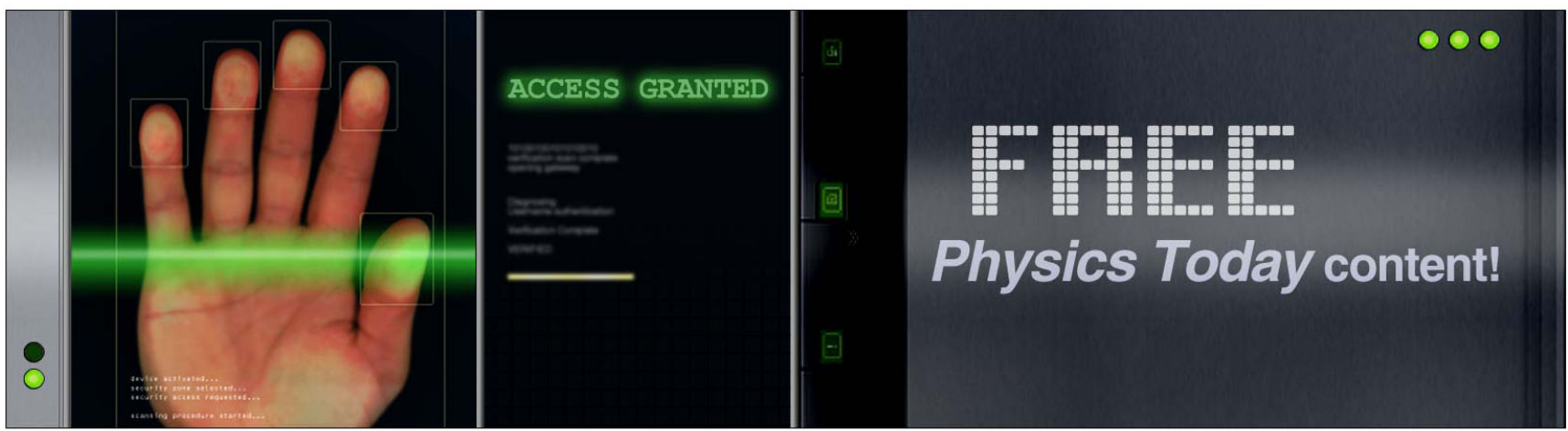


\title{
Low threshold, room-temperature microdisk lasers in the blue spectral range
}

\author{
Igor Aharonovich, ${ }^{1,2, a), b)}$ Alexander Woolf, ${ }^{1, a)}$ Kasey J. Russell, ${ }^{1, a)}$ Tongtong Zhu, ${ }^{3}$ \\ Nan Niu, ${ }^{1}$ Menno J. Kappers, ${ }^{3}$ Rachel A. Oliver, ${ }^{3}$ and Evelyn L. Hu ${ }^{1}$ \\ ${ }^{1}$ School of Engineering and Applied Sciences, Harvard University, Cambridge, Massachusetts 02138, USA \\ ${ }^{2}$ School of Physics and Advanced Materials, University of Technology Sydney, Ultimo 2007, \\ New South Wales, 2007, Australia \\ ${ }^{3}$ Department of Materials Science and Metallurgy, University of Cambridge, Pembroke Street, \\ Cambridge CB2 3QZ, United Kingdom
}

(Received 21 February 2013; accepted 25 June 2013; published online 10 July 2013)

\begin{abstract}
InGaN-based active layers within microcavity resonators offer the potential of low threshold lasers in the blue spectral range. Here, we demonstrate optically pumped, room temperature lasing in high quality factor GaN microdisk cavities, containing InGaN quantum dots (QDs) with thresholds as low as $0.28 \mathrm{~mJ} / \mathrm{cm}^{2}$. The demonstration of lasing action from GaN microdisk cavities with QDs in the active layer, provides a critical step for the nitrides in realizing low threshold photonic devices with efficient coupling between QDs and an optical cavity (C) 2013 AIP Publishing LLC.

[http://dx.doi.org/10.1063/1.4813471]
\end{abstract}

High quality factor $(Q)$, small volume microcavities in GaN-based materials offer the prospect of low threshold lasers in the UV to visible range. Quantum well (QW) active regions have been incorporated into microdisks, ${ }^{1-7}$ microrings, ${ }^{8}$ and photonic crystal structures. ${ }^{9,10}$ In general, the room-temperature thresholds of the structures have been in the range of a few $\mathrm{mJ} / \mathrm{cm}^{2}$ (for pulsed measurements), ${ }^{11}$ or a few hundred $\mathrm{kW} / \mathrm{cm}^{2}(\mathrm{CW}){ }^{6,12}$ This work describes the incorporation of quantum dot (QD)-containing active regions within high $Q(\sim 6000)$ microdisk cavities. Lasing was achieved for 1 micron diameter microdisks incorporating 3 layers of QDs, at threshold energies as low as $0.28 \mathrm{~mJ} / \mathrm{cm}^{2}$. Our experiments took place with the concurrent evaluation of four sample structures which allowed us to probe the influence of QD density and cavity design on the lasing threshold. These results represent important progress in realizing the potential of quantum dot active layers in GaN-based lasers. The spatial localization and distinctive density of states for QDs promise greater immunity to material defects and intrinsically lower threshold operation. In addition, the concurrent comparison of different materials and cavity structures will allow us to better understand the critical factors that determine the onset of lasing.

A schematic illustration of the sample structure is shown in Figs. 1(a), and 1(c) summarizes the properties of the four materials used in this work. Briefly, the top disk membrane has a thickness of either $120 \mathrm{~nm}$ or $200 \mathrm{~nm}$ and encapsulates either one or three layers of $\operatorname{In}_{0.2} \mathrm{Ga}_{0.8} \mathrm{~N}$ with each layer containing QDs with an approximate areal density of $1 \times 10^{10} \mathrm{~cm}^{-2}$ based on atomic force microscopy studies of QD epilayers grown contemporaneously. ${ }^{13,14}$ It should be noted that the InGaN layers contain both QDs and an inhomogeneous QW. The implications of this structure will be discussed later.

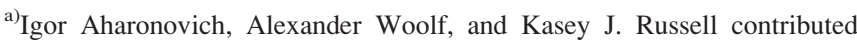
equally to this work.

b) Author to whom correspondence should be addressed. Electronic mail: igor.aharonovich@uts.edu.au.
}

The pedestal of the disk is an $\operatorname{In}_{\mathrm{x}} \mathrm{Ga}_{1-\mathrm{x}} \mathrm{N} / \mathrm{In}_{\mathrm{y}} \mathrm{Ga}_{1-\mathrm{y}} \mathrm{N}$ sacrificial superlattice (SSL, $x=5.1 \%, y=6.5 \%$ In) which is grown on an n-doped GaN pseudo-substrate and is capped by a thin $\sim 10 \mathrm{~nm}$ GaN layer, followed by an $\mathrm{Al}_{0.2} \mathrm{Ga}_{0.8} \mathrm{~N}$ etch stop layer. ${ }^{13,15}$ The samples have a similar surface pit density, indicating that they have a similar threading dislocation density. This allows us to make the first-order assumption that variations in the performance of the cavities or the lasers are not due to variations in principal defect densities in the starting materials.

The microdisks were fabricated by dispersing $1 \mu \mathrm{m}$ silica beads onto the samples which served as etch hard masks. ${ }^{12,16}$ The samples were then etched using a $500 \mathrm{~W}$ inductively coupled plasma reactive ion etching process in an argon/chlorine environment, with a flow rate of $25 / 25$ standard cubic centimeters per minute, respectively. The silica beads were removed by immersing the samples in water in an ultrasonic bath for $5 \mathrm{~min}$. A metal film (Ti/Au), which serves as the cathode for the photo electrochemical (PEC) etching, was then deposited using an e-beam evaporator. Finally, to optically isolate the membrane layer, the microdisks were undercut using PEC etching. The disks were immersed in $0.004 \mathrm{M} \mathrm{HCl}$ solution and illuminated using a $1000 \mathrm{~W}$ Xe lamp for $18 \mathrm{~min}$. A GaN filter was used to generate electron-hole pairs only in the sacrificial post pedestal layer (below the GaN bandgap), thus etching it away and creating the undercut. Fig. 1(d) shows a scanning electron microscope micrograph of a fabricated microdisk from sample A. The vertical confinement is achieved by undercutting the structure and isolating the periphery of the microdisk in air.

The optical properties of the microdisk resonators were investigated using a frequency doubled, pulsed, titaniumsapphire laser emitting at $380 \mathrm{~nm}(76 \mathrm{MHz}$ repetition rate, $200 \mathrm{ps}$ pulse duration) through a high (0.95) numerical aperture (NA) objective normal to the surface of the microdisk. The luminescence from the microdisks was collected through the same objective. Photoluminescence (PL) spectra recorded at room temperature from $1 \mu \mathrm{m}$ diameter microdisks show 


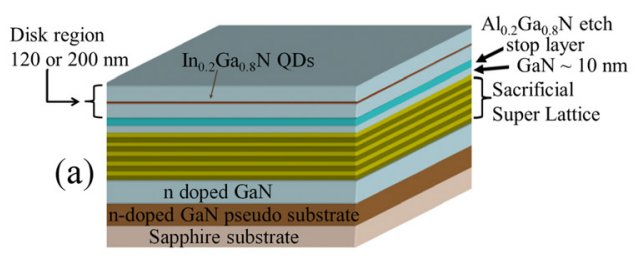

(c)

\begin{tabular}{|c|c|c|c|l|}
\hline Sample & $\begin{array}{c}\text { QDs } \\
\text { layers }\end{array}$ & $\begin{array}{c}\text { Membrane } \\
\text { thickness (nm) }\end{array}$ & $\begin{array}{l}\text { Peak emission } \\
\text { wavelength }\end{array}$ & $\begin{array}{l}\text { Surface pit } \\
\text { density }\left(\mathrm{cm}^{-2}\right)\end{array}$ \\
\hline A & 3 & 200 & $439 \pm 2 \mathrm{~nm}$ & $(5 \pm 0.4) \times 10^{8}$ \\
\hline B & 3 & 120 & $447 \pm 8 \mathrm{~nm}$ & $(5 \pm 0.6) \times 10^{8}$ \\
\hline C & 1 & 200 & $454 \pm 5 \mathrm{~nm}$ & $(4 \pm 0.6) \times 10^{8}$ \\
\hline $\mathrm{D}$ & 1 & 120 & $467 \pm 9 \mathrm{~nm}$ & $(4 \pm 0.5) \times 10^{8}$ \\
\hline
\end{tabular}

(b)
Deposit $\mathrm{SiO}_{2}$ beads which Reactive Ion etch, $\mathrm{Ar} / \mathrm{Cl}_{2}$ $\begin{array}{lll}\begin{array}{l}\text { Deposit } \mathrm{SiO}_{2} \text { beads which } \\ \text { serve as a hard mask }\end{array} & \begin{array}{l}\text { Reactive Ion etch, } \mathrm{Ar} / \mathrm{Cl}_{2} \\ \text { chemistry } \downarrow \downarrow \downarrow\end{array} & \text { Beads removal }\end{array}$ chemistry $\downarrow \downarrow$
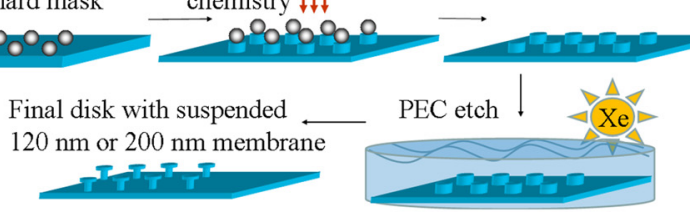

(d)

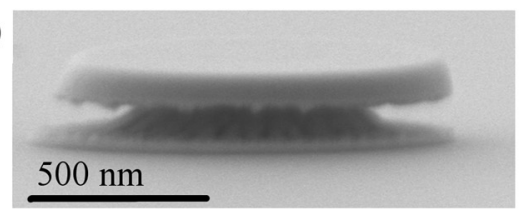

FIG. 1. (a) A schematic illustration of the sample structure. The disk region has a thickness of 120 or $200 \mathrm{~nm}$ with either one or three embedded QD layers. (c) Properties of the four investigated samples. (b) Schematic illustration of the procedure used to fabricate the microdisks. (d) Scanning electron microscope images (top and side view) of the $1 \mu \mathrm{m}$ diameter microdisk cavity.

whispering gallery modes (WGMs), modulating the broad emission from the QD-containing layers (Fig. 2(a)). Figure 2(b) shows a high resolution spectrum of the transverse electric $\left(\mathrm{TE}_{1,11}\right)$ mode at $\sim 475.8 \mathrm{~nm}$ from sample A. This mode exhibits a splitting between the two normally-degenerate counterpropagating whispering gallery modes that is likely due to slight imperfections in fabrication that destroy the rotational symmetry of the disk. These two modes are fitted with Lorentzian functions, as indicated with red lines in Fig. 2(b). The $\mathrm{Q}$ values for each resonance were determined by calculating $\lambda_{\text {cav }} / \Delta \lambda_{\text {cav }}$, where $\lambda_{\text {cav }}$ is the cavity mode wavelength and $\Delta \lambda_{\text {cav }}$ is the full width at half maximum (FWHM) of the mode. The highest measured $Q$ for $1 \mu$ m-diameter disks from sample A, $Q \sim 6600$, is one of the highest $Q s$ reported from GaNbased microdisks. ${ }^{17}$

Lasing behavior in microdisks is observed through the dependence of the PL emission intensity and mode linewidth on excitation power, as is shown in Fig. 3(a). At excitation powers below the lasing threshold, multiple WGMs are observed. As the excitation power is increased through the lasing threshold, the intensity of a single mode increases abruptly and at higher powers dominates the emission. This behavior is indicative of a transition from spontaneous emission to lasing. The lasing mode in this case was determined to be the $\mathrm{TE}_{1,13}$ mode based on finite difference time domain (FDTD) simulations. ${ }^{18}$ A plot of the output intensity versus input power for microdisks from sample A (blue triangles) and sample B (red circles) is shown in Fig. 3(b), with clear thresholds at $\sim 0.28 \mathrm{~mJ} / \mathrm{cm}^{2}$ and $0.63 \mathrm{~mJ} / \mathrm{cm}^{2}$, respectively. The lasing threshold was determined as the intersection of the horizontal axis and a linear fit to the higher-power region of the data. The same data plotted on a log-log scale (top right inset, Fig. 3(c)) clearly show all three regimes of operation: spontaneous emission, amplified spontaneous emission, and laser oscillation. ${ }^{19}$ In addition, we observed a pronounced narrowing of the lasing mode as the excitation power was increased through the lasing threshold, signifying the increased temporal coherence of emission in the lasing regime (Fig. 3(c)). Taken together, these data unambiguously demonstrate the achievement of lasing behavior in our devices. The bottom left inset of Fig. 3(c) shows the optical image of the microdisk laser above lasing threshold recorded using a CCD camera.

A comparison among samples A-D provides an indication of the most critical factors affecting the lasing behavior of these devices. All microdisks included in this analysis exhibited pronounced modes in the PL spectra with a minimum $Q$ of 1000 . Of the four samples investigated in this study, only samples A and B exhibited lasing behavior. Sample A showed lower threshold lasing because it exhibits both high $Q$ and it has three layers of QDs that contribute to the emission gain. The other samples either have thinner membranes, which FDTD simulations suggest should lead to lower maximum $Q(\sim 18000)$ for $120 \mathrm{~nm}$ membrane versus $\mathrm{Q} \sim 25000$ for the $200 \mathrm{~nm}$ membrane, or have fewer layers of QDs. These variations in theoretical and actually-observed $Q$ 's of the structure and in density of the gain medium provide an excellent probe of the critical parameters in material and structure that give rise to low threshold lasing.

For all disks that achieved lasing, values were extracted for the threshold power, mode wavelength, and maximum cavity $Q$ measured at low excitation power (Fig. 4). On
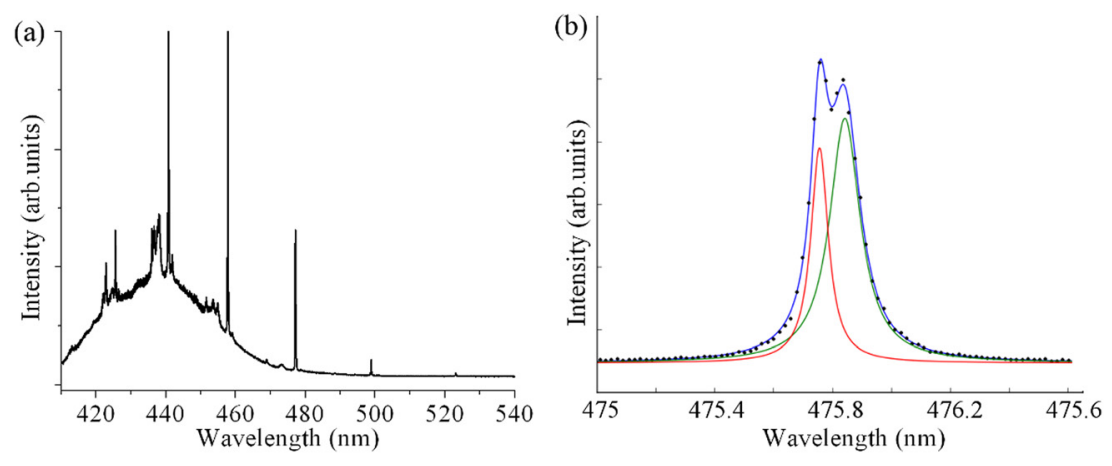

FIG. 2. (a) Optical characterization of a microdisk made of material A. PL spectrum recorded using $380 \mathrm{~nm}$ excitation wavelength, showing predominantly first order WGMs decorating the broad QD-related emission. (b) High resolution PL spectrum of a high $\mathrm{Q}$ mode with $\mathrm{Q} \sim 6600$. Green and red lines are fits to a Lorentzian. 

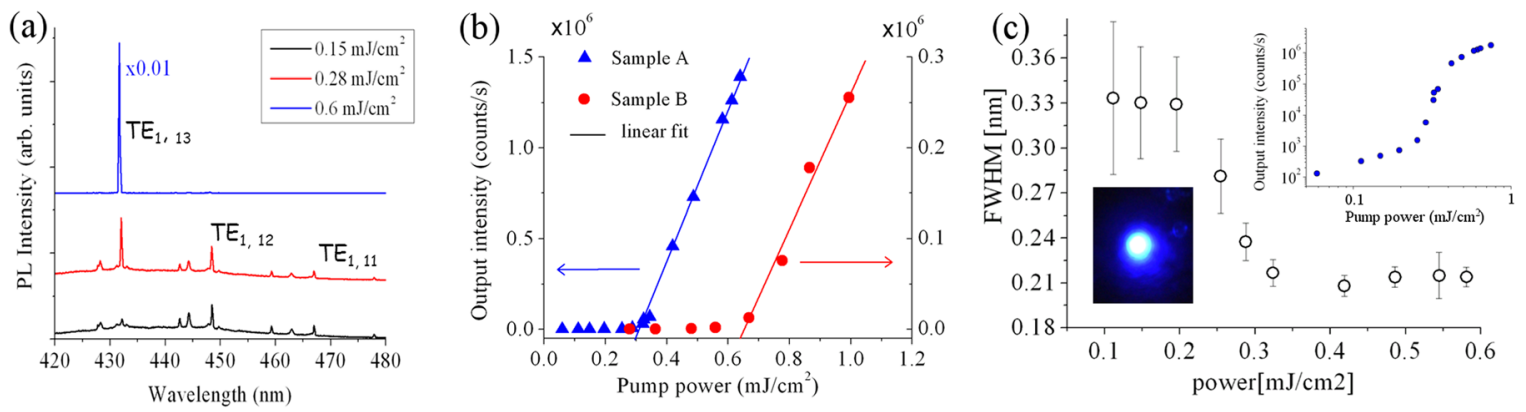

FIG. 3. (a) PL spectra as a function of excitation power recorded from a $1 \mu \mathrm{m}$ size microdisk cavity (Sample A). (b) Optical output power of the microdisk laser as a function of excitation power for sample A (blue triangles) and B (red circles). A clear lasing threshold is observed at $0.28 \mathrm{~mJ} / \mathrm{cm}^{2}$ and $0.63 \mathrm{~mJ} / \mathrm{cm}^{2}$ for samples A and B, respectively. The lines are a linear fit to the data above threshold. (c) Linewidth of the lasing mode plotted as a function of excitation power for sample A. The reduction of the linewidth is in accord with lasing behavior. Inset top right, same data as in (b) replotted on a logarithmic scale. Inset bottom left, an optical image of the microdisk laser above threshold recorded using a CCD camera.

sample A, 6 of 10 disks achieved lasing, with a threshold that varied from 0.28 to $0.92 \mathrm{~mJ} / \mathrm{cm}^{2}$. Sample B, which had the same number of layers of QDs as sample A but a thinner membrane, achieved lasing in only 3 of 10 disks, with thresholds in the range $0.4-1.1 \mathrm{~mJ} / \mathrm{cm}^{2}$. The typical $Q$ of modes from microdisks on sample B was lower than on sample A (Fig 4(a)). This trend is consistent with FDTD simulations, although in both samples the measured $Q$ is more than an order of magnitude lower than the theoretical limit. This latter is not unusual, since the simulations do not account for imperfections in the material or in the fabrication of the microdisks.

In samples $\mathrm{C}$ and $\mathrm{D}$, which only contained a single layer of QDs, lasing was not observed on any disks under the excitation powers available in our experimental setup. In addition, the $Q$ of these cavities was comparable to or lower than cavities from samples A and B, respectively. These observations suggest that re-absorption within the $\mathrm{InGaN}$ layers themselves is not the dominant factor limiting $Q$ in these structures and that, at least under pulsed excitation at room temperature, multiple QD layers are necessary to supply sufficient gain to compensate losses. The importance of the areal density of quantum dots, and how it limits the modal gain of the active layer has been earlier noted for InGaAs-based quantum dot lasers. ${ }^{20}$ Indeed, larger cavity, in-plane lasers with InGaN quantum dot active material have employed 8 to 10 QD layers. ${ }^{5,21,22}$ We also note that no clear dependence is observed between the lasing threshold and the $Q$ of the microdisk. Low InGaN re-absorption is consistent with the low number of QDs coupled to a mode: each $1 \mu$ m-diameter disk contains approximately 100 QDs per layer at a QD density of $\sim 10^{10} \mathrm{~cm}^{-2}$, and of these, it is likely that fewer than 10 are in spatial and spectral resonance with a WGM.

Because re-absorption is sufficiently low in these devices, modes are visible across the entire gain spectrum and not only at the low-energy side of the spectrum (as is typical for $\mathrm{GaN}$ microdisks with quantum well active region ${ }^{3,23}$ ). In fact, in our devices the lasing mode was consistently located at wavelengths shorter than $430 \mathrm{~nm}$, on the high-energy side of the broad QD emission spectrum (Fig. 4(b)). This dramatic blue-shift of the lasing wavelength for pulsed, rather than CW optical excitation has been observed previously in InGaAs QD microdisk lasers. ${ }^{24}$ The reasons for this behavior will be explored further, but may relate to a differential change in radiative lifetimes within a cavity environment with a high instantaneous charged carrier background. The complex interplay between the energies of the lasing mode and the QD gain spectrum is evident in the variation of lasing threshold with wavelength (Fig. 4(b)). The threshold decreases at longer wavelengths as the overlap with the QD emission spectrum increases. Nevertheless, lasing from a mode at the center of the QD emission spectrum was not observed.

Several aspects of this comparison highlight the need for further understanding of the interplay of active layer composition and cavity structure in determining the lasing thresholds of the resulting devices. Further exploration is needed on the optimal InGaN QD material structure, the influence of background defects in the materials, the wavelength-dependence of the QD radiative lifetimes and efficiencies, as well as optimal cavity designs. In addition, our gain medium consists of InGaN layers which contain not only QDs but also an inhomogeneous ("patchy") quantum well layer. A atomic force microscope data indicating that the QDs may be located not only on top of the QW layer (similar to the case of Stranski-Krastanov growth in, for example, InAs/GaAs, where QDs sit on top a wetting layer) but also in the "patches" between quantum well regions. The sample exhibits both QD-like and QW-like emission. QD emission has been confirmed for these samples at the wavelengths at which lasing is observed using low temperature microPL. ${ }^{13}$
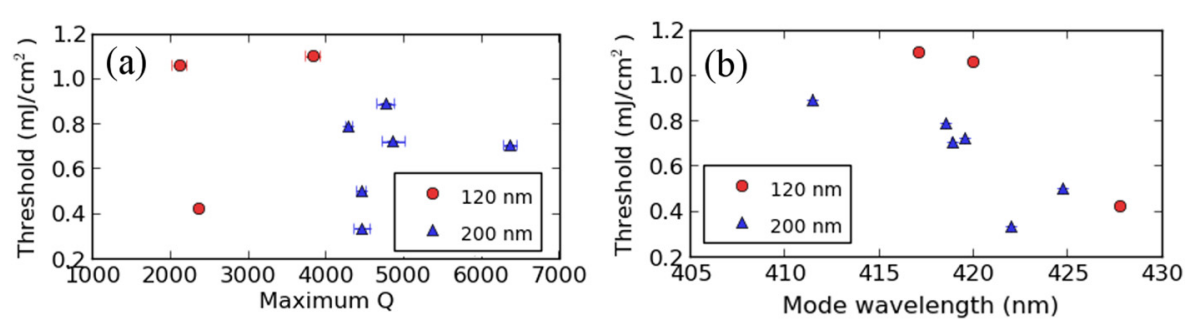

FIG. 4. (a) Lasing threshold is plotted as a function of maximum $\mathrm{Q}$ recorded from the same microdisk for sample A (blue triangles) and sample B (red circles). (b) Lasing threshold is plotted as a function of a lasing wavelength for sample A (blue triangles) and sample B (red circles). 
To summarize, we have characterized a set of microdisk lasers with quantum dot-containing active layers. Of the four samples investigated in this study, only samples A and B, containing 3 layers of QDs exhibited lasing behavior with thresholds as low as $0.28 \mathrm{~mJ} / \mathrm{cm}^{2}$ under pulsed excitation at room temperature. Our results suggest the critical importance of sufficiently high QD areal density. Although the lasing thresholds of these devices are exceptionally low, the sparser areal density of the quantum dot gain material, compared with quantum wells, may explain the yet lower threshold of $300 \mathrm{~W} / \mathrm{cm}^{2}$ reported by Tamboli et al. ${ }^{3}$ for a QW microdisk laser under continuous wavelength excitation at room temperature. A better strategic design of the InGaN QD active layer material, matched to a smaller mode volume cavity (e.g., a photonic crystal cavity) may result in still lower values of lasing thresholds. We believe that our studies are important not only for the efficient lasing performance demonstrated but also because of the important insights gained on the relative impact on lasing of the materials composition and structure, matched to the microdisk design and fabrication.

The authors acknowledge Haitham A. R. El-Ella for assistance with the sample growth. This work was supported in part by the Engineering and Physical Sciences Research Council (Award No. EP/H047816/1) and by the NSF Materials World Network (Award No. 1008480). This work was enabled by facilities available at the Center for Nanoscale Systems, a member of the National Nanotechnology Infrastructure Network (NNIN) which is supported by the National Science Foundation under NSF Award No. ECS-0335765. Dr. Aharonovich is the recipient of an Australian Research Council Discovery Early Career Research Award (Project No. DE130100592).

\footnotetext{
${ }^{1}$ M. Khajavikhan, A. Simic, M. Katz, J. H. Lee, B. Slutsky, A. Mizrahi, V. Lomakin, and Y. Fainman, Nature 482(7384), 204-207 (2012).

${ }^{2}$ E. Feltin, G. Christmann, R. Butte, J. F. Carlin, M. Mosca, and N. Grandjean, Appl. Phys. Lett. 89(7), 071107 (2006).
}

${ }^{3}$ A. C. Tamboli, E. D. Haberer, R. Sharma, K. H. Lee, S. Nakamura, and E. L. Hu, Nature Photon. 1(1), 61-64 (2007).

${ }^{4}$ C. H. Lin, J. Y. Wang, C. Y. Chen, K. C. Shen, D. M. Yeh, Y. W. Kiang, and C. C. Yang, Nanotechnology 22(2), 025201 (2011).

${ }^{5}$ K. Tachibana, T. Someya, Y. Arakawa, R. Werner, and A. Forchel, Appl. Phys. Lett. 75(17), 2605-2607 (1999).

${ }^{6}$ D. Simeonov, E. Feltin, A. Altoukhov, A. Castiglia, J. F. Carlin, R. Butte, and N. Grandjean, Appl. Phys. Lett. 92(17), 171102 (2008).

${ }^{7}$ C.-C. Chen, M. H. Shih, Y.-C. Yang, and H.-C. Kuo, Appl. Phys. Lett. 96(15), 151115 (2010).

${ }^{8}$ K. C. Zeng, L. Dai, J. Y. Lin, and H. X. Jiang, Appl. Phys. Lett. 75(17), 2563-2565 (1999).

${ }^{9}$ N. V. Trivino, G. Rossbach, U. Dharanipathy, J. Levrat, A. Castiglia, J. F. Carlin, K. A. Atlasov, R. Butte, R. Houdre, and N. Grandjean, Appl. Phys. Lett. 100(7), 071103 (2012).

${ }^{10}$ Y. S. Choi, K. Hennessy, R. Sharma, E. Haberer, Y. Gao, S. P. DenBaars, S. Nakamura, E. L. Hu, and C. Meier, Appl. Phys. Lett. 87(24), 243101 (2005).

${ }^{11}$ T. T. Wu, P. H. Weng, Y. J. Hou, and T. C. Lu, Appl. Phys. Lett. 99(22), 221105 (2011).

${ }^{12}$ E. D. Haberer, R. Sharma, C. Meier, A. R. Stonas, S. Nakamura, S. P. DenBaars, and E. L. Hu, Appl. Phys. Lett. 85(22), 5179-5181 (2004).

${ }^{13}$ T. T. Zhu, H. A. R. El-Ella, B. Reid, M. J. Holmes, R. A. Taylor, M. J. Kappers, and R. A. Oliver, J. Cryst. Growth 338(1), 262-266 (2012).

${ }^{14}$ R. A. Oliver, M. J. Kappers, J. Sumner, R. Datta, and C. J. Humphreys, J. Cryst. Growth 289(2), 506-514 (2006).

${ }^{15}$ R. A. Oliver, G. A. D. Briggs, M. J. Kappers, C. J. Humphreys, S. Yasin, J. H. Rice, J. D. Smith, and R. A. Taylor, Appl. Phys. Lett. 83(4), 755-757 (2003).

${ }^{16}$ H. A. R. El-Ella, F. Rol, M. J. Kappers, K. J. Russell, E. L. Hu, and R. A. Oliver, Appl. Phys. Lett. 98(13), 131909 (2011).

${ }^{17}$ T. Guillet, M. Mexis, S. Sergent, D. Neel, S. Rennesson, C. Brimont, T. Bretagnon, B. Gil, D. Sam-Giao, B. Gayral, F. Semond, M. Leroux, S. David, X. Checoury, and P. Boucaud, Phys. Status Solidi B 249(3), 449-454 (2012).

${ }^{18}$ S. C. Hagness, D. Rafizadeh, S. T. Ho, and A. Taflove, J. Lightwave Technol. 15(11), 2154-2165 (1997).

${ }^{19}$ A. E. Siegman, Lasers (University Science Books, Mill Valley, CA, 1986).

${ }^{20}$ D. Bimberg, N. Kirstaedter, N. N. Ledentsov, Z. I. Alferov, P. S. Kopev, and V. M. Ustinov, IEEE J. Sel. Top. Quantum Electron. 3(2), 196-205 (1997).

${ }^{21}$ A. Banerjee, T. Frost, E. Stark, and P. Bhattacharya, Appl. Phys. Lett. 101(4), 041108 (2012).

${ }^{22}$ M. Zhang, A. Banerjee, C.-S. Lee, J. M. Hinckley, and P. Bhattacharya, Appl. Phys. Lett. 98(22), 221104 (2011).

${ }^{23}$ S. Chang, N. B. Rex, R. K. Chang, G. Chong, and L. J. Guido, Appl. Phys. Lett. 75(2), 166-168 (1999).

${ }^{24}$ D. Young, PhD Thesis (University of California, Santa Barbara, 2002). 